Service social

\title{
Recension des écrits sur le soutien à domicile : la personne âgée et les ressources communautaires, des acteurs oubliés
}

\author{
Aline Vézina, Jean Vézina et Caroline Tard
}

Volume 43, numéro 1, 1994

Maintien à domicile

URI : https://id.erudit.org/iderudit/706643ar

DOI : https://doi.org/10.7202/706643ar

Aller au sommaire du numéro

Éditeur(s)

École de service social de l'Université Laval

ISSN

1708-1734 (numérique)

Découvrir la revue

Citer cet article

Vézina, A., Vézina, J. \& Tard, C. (1994). Recension des écrits sur le soutien à domicile : la personne âgée et les ressources communautaires, des acteurs oubliés. Service social, 43(1), 67-85. https://doi.org/10.7202/706643ar
Résumé de l'article

Le présent article constitue à la fois un reflet des principales conclusions émises par les auteurs répertoriés au cours d'une récente recension des écrits et une occasion de réfléchir sur le soutien des personnes âgées en contexte québécois. Dans une approche plus descriptive, les auteurs présentent les études qui abordent la contribution des acteurs au soutien à domicile des personnes âgées ainsi que les théories, modèles et typologies qui tentent d'expliquer les relations entre les acteurs. En guise de conclusion, certains constats théoriques, méthodologiques et politiques sont discutés. 


\title{
Recension des écrits sur le soutien à domicile : la personne âgée et les ressources communautaires, des acteurs oubliés
}

\author{
Aline VÉZINA \\ Professeure, École de service social, Université Laval \\ Jean VÉZINA \\ Professeur, École de psychologie, Université Laval
}

Caroline TARD

Professionnelle de recherche, Centre de recherche sur les services communautaires, Université Laval

\section{CONTEXTE DE LA RECENSION}

Cette recension des écrits ${ }^{1} s^{\prime}$ inscrit dans la foulée des travaux entourant la problématique du partage des responsabilités entre la famille et l'État, dans le cadre du soutien à domicile des personnes âgées. Elle repose sur deux constats importants, soit :

1) I'accroissement des demandes adressées aux différents réseaux $d^{\prime}$ aide et de soins (formel, informel et communautaire) provoqué par le nombre de plus en plus élevé de personnes âgées et

1. Cette recension des écrits a été faite grâce à une subvention du Conseil québécois de la recherche sociale (RS 1915 091). 
2) les réorientations des politiques sociales québécoises affectant les personnes âgées et les services qui leur seront fournis dans le cadre d'un partenariat recherché.

La question des politiques sociales affectant les personnes âgées et des services qui leur seront fournis a fait l'objet de plusieurs enquêtes nationales (Commission Rochon, 1987 ; Rapport Brunet, 1987 ; Santé et Bien-être social Canada, 1986). Cela témoigne d'une préoccupation politique de mieux orienter les services en fonction de la capacité de payer de l'État. À la suite de ces enquêtes, des énoncés de politique (Commission d'enquête sur la santé et les services sociaux, 1988; Conseil consultatif national sur le troisième âge, 1989 et 1990) ainsi que, plus récemment, Une réforme axée sur le citoyen (ministère de la Santé et des Services sociaux, 1990) et La politique de la santé et du bienêtre parrainée par le ministre Marc-Yvan Côté (ministère de la Santé et des Services sociaux, 1992) ouvrent la porte à la mise en place d'un nouveau partenariat fondé sur le potentiel du réseau naturel et communautaire en accord avec le réseau socio-sanitaire officiel. Le but avoué de cette recherche d'un nouveau partenariat est d'assurer, le plus longtemps possible, le soutien de la personne âgée dans son milieu naturel et ainsi diminuer le recours à des services de plus en plus coûteux, tels que l'hébergement public, les urgences et les hôpitaux (Association pour la santé publique du Québec, 1988).

\section{OBJECTIF, ORIGINALITÉ ET RETOMBÉES DE LA RECENSION DES ÉCRITS}

L'objectif de la présente recension des écrits est d'étudier la dynamique des relations entre les acteurs impliqués dans le soutien à domicile des personnes âgées. Les quatre acteurs étudiés sont la personne âgée ellemême, son réseau informel ou naturel de soutien (famille, amis et voisins), le réseau communautaire et le réseau formel, c'est-à-dire les services fournis par l'État. Dans le cadre de cette recension, la dynamique des relations se définit comme l'ensemble des changements qui peuvent survenir dans les contributions et les relations entre les quatre acteurs, au rythme des transformations personnelles, familiales, sociales, politiques et économiques.

L'originalité et la pertinence de cette recherche bibliographique reposent tout $\mathrm{d}^{\prime}$ abord sur un constat théorique et empirique. En effet, un survol des écrits sur le soutien à domicile des aînés permet de relever un nombre de plus en plus grand d'écrits concernant les facteurs qui prédisposent à I'hébergement des personnes âgées (Béland, 1982; 
Harper et Lund, 1990; Pruchno, Michaels et Potashnik, 1990), le mythe du désengagement de la famille auprès de ses membres âgés (Garant et Bolduc, 1990 ; Roy, Vézina et Paradis, 1992) et l'évaluation des ressources auprès des personnes âgées (Bolduc, Bélanger et Dery, 1990; Grandmaison et Gosselin, 1986 ; Joubert, Laberge, Fortin, Paradis et Desbiens, 1991). Cependant, la dynamique des relations entre les acteurs a été très peu étudiée.

L'originalité de la présente recension réside également dans le fait que celle-ci propose une vision "autonomiste» de la personne âgée en la considérant comme un acteur important de son propre soutien en milieu naturel. Elle veut éviter de considérer la condition des personnes vieillissantes strictement en termes de problèmes, de manques ou de déficits.

Les retombées possibles pour le monde de la pratique ajoutent à la pertinence de cette recension. En effet, en plus d'apporter un éclairage théorique, cette recension des écrits peut certainement contribuer à une meilleure concertation dans la mise en place de services, en tenant compte des forces et des limites de chacun des acteurs.

Le présent article constitue à la fois un reflet des principales conclusions émises par les auteurs recensés et une occasion de réfléchir sur le soutien à domicile des personnes âgées en contexte québécois. Dans une approche plus descriptive, les études qui abordent la contribution des acteurs au soutien à domicile des personnes âgées ainsi que les théories, modèles et typologies qui tentent d'expliquer les relations qui s'établissent entre les acteurs seront présentés. En guise de conclusion, certains constats théoriques, méthodologiques et politiques seront discutés.

\section{LA CONTRIBUTION DES ACTEURS}

D'entrée de jeu, une remarque de nature quantitative donne le ton à l'ensemble de la recension des écrits qui, rappelons-le, porte sur la dynamique des relations entre les acteurs engagés dans le soutien à domicile des personnes âgées ${ }^{2}$. Ainsi, parmi les 51 documents retenus, la presque totalité des ouvrages (39) explorent les relations entre le réseau formel et le réseau informel. Quatre textes (Chappell, 1987;

2. Pour une présentation complète de la recension des écrits ainsi que de la méthodologie utilisée, consulter A. Vézina, J. Vézina et C. Tard (1993), Dynamique des relations des quatre acteurs impliqués dans le soutien des personnes âgées à domicile. Centre de recherche sur les services communautaires, Université Laval. 
Jobert, 1988; McFarland, 1991 ; Seltzer, Ivry et Litchfield, 1987) traitent des relations entre le réseau informel, le réseau formel et la personne âgée. Les relations impliquant le réseau informel, le réseau formel et les ressources communautaires font l'objet de trois textes (Jutras et Veilleux, 1989; Litwak, 1985 ; Litwak, Messeri et Silverstein, 1990). Deux documents (Hughes et Guihan, 1990; Panet-Raymond et Vandal, 1991) abordent les relations entre le réseau formel et les ressources communautaires. Finalement, les relations entre les quatre acteurs sont présentées dans trois ouvrages (Cantor, 1991; Hokenstad et Johansson, 1990 ; Roy et al., 1992).

Quelles tâches accomplissent la famille et les amis auprès de la personne âgée vivant dans son milieu naturel? Quelles sont la nature et la quantité de l'aide apportée par le réseau formel et le réseau communautaire? Quelle est la contribution de la personne âgée à son propre soutien à domicile? Voici quelques questions auxquelles certains auteurs tentent de répondre.

D'après l'étude de Wisendale (1983) menée auprès d'un peu plus de 1800 aînés, il existe peu de différences dans la division des tâches entre le réseau formel et informel. Pour sa part, Schirm (1987) observe que les soins personnels des aînés en perte d'autonomie (aider à manger, à sortir du lit ou du fauteuil, à se vêtir, à faire sa toilette, fournir les soins en cas $d^{\prime}$ incontinence) et certaines tâches de la vie domestique (préparation des repas, lessive, ménage) sont partagés entre le réseau informel et le réseau formel. De façon générale, les aidants informels, généralement les membres de la famille, sont plus susceptibles de fournir de l'assistance pour remplir toutes les tâches domestiques et pour donner des médicaments. Les intervenants des services publics, quant à eux, donnent les bains et gèrent la supervision et la formation aux soins.

Dans une étude menée auprès d'une centaine de personnes âgées, McFarland (1991) a analysé l'effet de la prestation des services publics sur la contribution des membres du réseau informel en ce qui regarde, notamment, le temps passé à apporter des soins et de l'aide. Elle note que lorsque l'État fournit des services, les membres du réseau informel passent moins de temps à exécuter les tâches de la vie domestique (transport, commissions, ménage et préparation des repas), qui sont des tâches sans incidence émotionnelle et sans entraînement professionnel. Pour les personnes les plus âgées, de sexe masculin et ayant des limitations fonctionnelles importantes, le réseau informel continue de fournir le même nombre d'heures qu'avant l'introduction des services publics. Cette dernière conclusion rejoint les résultats présentés par Roy et al. (1992). 
Les résultats de l'étude de Jutras et Veilleux (1989) révèlent que l'assistance du réseau formel se résume à la consultation des professionnels de la santé, les familles fournissant l'essentiel de l'aide aux aînés. La contribution des organismes communautaires au soutien à domicile des personnes âgées serait, somme toute, selon ces auteures, inexistante.

La contribution des organismes communautaires a aussi été étudiée par Panet-Raymond et Vandal (1991). Selon ces auteurs, les organismes communautaires fournissent des services complémentaires au réseau officiel des CLSC et des centres de jour; ils offrent aussi des services spécifiques qui ont un caractère plus socio-affectif. Ils font également office de relais ou d'intermédiaires entre les services publics et les réseaux informels des personnes âgées.

L'étude des textes répertoriés montre que la contribution des acteurs au soutien de la personne âgée dans son milieu naturel est susceptible de se modifier en fonction de plusieurs facteurs.

\section{Les facteurs liés à la contribution des acteurs}

Les principaux facteurs liés à la contribution des acteurs dans le soutien à domicile des personnes âgées sont 1) l'âge de l'aîné, 2) le fait de vivre seul ou pas, 3) le niveau d'autonomie ou les incapacités fonctionnelles de l'individu âgé et finalement 4) la disponibilité du réseau naturel eu égard aux besoins de la personne âgée.

À la suite d'une recension d'écrits, Soldo et al. (1989) rapportent qu'un consensus existe sur le fait que l'âge de l'aîné est l'un des deux facteurs associés à la contribution des réseaux formel et informel. Les arrangements de vie constituent le second facteur. Ainsi, selon ces auteurs, les études recensées montrent qu'un âge avancé et le fait de vivre seul augmentent la probabilité de recevoir de l'aide de la part des réseaux formel et informel. Les résultats de Chappell et Blandford (1991) vont dans le même sens.

Le niveau d'autonomie ou les incapacités fonctionnelles de la personne âgée représentent un autre facteur qui détermine le recours à l'aide et aux soins du réseau formel, en plus du soutien apporté par le réseau naturel (Caro, 1986; Chappell, 1987 ; Chappell et Blandford, 1991 ; Horowitz, 1985 ; Soldo et al., 1989 ; Stoller, 1989).

D'après les études citées dans Soldo et al. (1989), près de $60 \%$ des personnes âgées, même en très sérieuse perte $d^{\prime}$ autonomie, reçoivent I'assistance des membres de leur réseau naturel. Un mélange $\mathrm{d}^{\prime}$ aidants formels et informels, résultant en des réseaux d'assistance plus étendus et diversifiés, apparaît lorsque les besoins de la personne âgée augmentent. Dans l'étude longitudinale de la dyade aidant/aidé 
de Stoller (1989), l'incapacité fonctionnelle constitue le facteur qui prédispose le plus au recours aux services publics. Ainsi, les aidants des personnes âgées qui souffrent de lourdes incapacités et qui ont besoin $d^{\prime}$ assistance pour les soins personnels sont les plus susceptibles d'utiliser les services formels.

Les résultats sont différents en ce qui concerne l'utilisation des ressources communautaires. Dans le cas de perte sérieuse d'autonomie, les groupes communautaires ne sont presque jamais reconnus comme ressources, par la personne âgée elle-même et par l'aidant principal (Roy et al., 1992). Les besoins d'une clientèle en perte sérieuse d'autonomie exigent une formation spécialisée que les bénévoles des organismes communautaires n'ont pas.

Le type d'incapacité (à court ou à long terme) est un autre facteur influençant le recours aux services de l'État (Chappell, 1987). Les personnes âgées qui ont des incapacités à court terme ont tendance à se tourner vers les membres de leur famille. Dans des conditions d'incapacités à long terme, les aînés font appel au réseau formel des soins.

La disponibilité du réseau naturel, en ce qui regarde les besoins de l'aîné, est également vue comme un facteur lié à la contribution du réseau formel (Factor, 1987; Horowitz, 1985 ; Soldo et al., 1989; Stoller, 1989; Wilcox et Taber, 1991). L'étude menée par Wilcox et Taber (1991) en Illinois, auprès de cent bénéficiaires d'un programme de soins à domicile, indique que la vaste majorité de ces personnes âgées reçoivent aussi de l'aide des membres du réseau informel. Les services publics sont requis au moment où la famille ne peut procurer tous les soins nécessaires. Chappell et Blandford (1991) notent que les personnes âgées recevant de l'aide et des soins, à la fois du réseau formel et informel, sont de deux types. Il y a les aînés qui souffrent de pertes d'autonomie variables (de légères à sévères), mais à qui le réseau informel ne peut fournir toute l'aide et tous les soins nécessaires. Il y a également les aînés qui éprouvent de grandes difficultés fonctionnelles tout en ayant un réseau naturel qui les soutient.

En résumé, après analyse des différents textes, il semble que les membres du réseau formel et informel accomplissent sensiblement les mêmes tâches auprès de la personne âgée, afin de la maintenir dans son milieu naturel. La contribution des différents acteurs varie en fonction des caractéristiques de la personnes âgée (âge, autonomie fonctionnelle et statut de cohabitation) et de la disponibilité des membres du réseau informel. Bien que la personne âgée en perte $d^{\prime}$ 'autonomie et vivant dans son milieu naturel soit le centre d'intérêt de tous les documents consultés pour cette recension, aucune étude ne décrit ou n'analyse sa contribution à son propre soutien à domicile. 
Tout au plus, certains auteurs émettent des souhaits pour que la personne âgée soit considérée comme un acteur participant aux décisions qui affectent l'aide et les soins qu'elle reçoit (Cantor, 1991; Jobert, 1988). La place du réseau communautaire dans le soutien à domicile des personnes âgées est aussi un des éléments très peu étudiés, I'essentiel des textes portant sur les contributions des réseaux formel et informel. Il est important de signaler que chez plusieurs auteurs américains les ressources communautaires, tout comme les services de I'État, se retrouvent sous le terme «réseau formel ». En faisant des ressources communautaires et des services de l'État un tout indifférencié, les auteurs éliminent la possibilité d'étudier les types de relations ou l'absence de relations qui peuvent s'établir entre ces deux acteurs qui se distinguent quant à leurs structures et à leurs missions par rapport au soutien à domicile des personnes âgées.

\section{LES RELATIONS ENTRE LES ACTEURS: THÉORIE, MODÈLE ET TYPOLOGIES}

Une théorie, un modèle et des typologies ont été proposés afin de décrire et de mieux comprendre les contributions et les relations entre les acteurs engagés dans le soutien à domicile de la personne âgée. Force est de constater une fois de plus que les contributions de la personne âgée et des ressources communautaires sont peu présentes, voire absentes chez plusieurs auteurs.

\section{La théorie des tâches spécifiques de Litwak}

La théorie des tâches spécifiques élaborée par Litwak (1985) est fréquemment retenue dans les écrits portant sur les relations entre les réseaux formel et informel d'aide et de soins aux personnes âgées (Horowitz, 1985). Garant et Bolduc (1990) en font d'ailleurs une excellente synthèse. Le postulat à la base de cette théorie est que les tâches accomplies par les deux types de réseaux sont dépendantes de la structure de chaque groupe. Ainsi, la structure du groupe primaire, c'està-dire du réseau informel, va de pair avec les dimensions des soins ne requérant pas de connaissances techniques, alors que la structure des organisations formelles va de pair avec les tâches nécessitant une connaissance technique, un savoir professionnel, spécialisé. La théorie des tâches spécifiques considère que les groupes primaires et les organisations formelles sont interdépendants et que, malgré leurs structures différentes, voire opposées, ils peuvent aider la personne âgée à se maintenir à 
domicile en coordonnant leurs efforts. En ce qui concerne les ressources communautaires, Litwak et al. (1990) indiquent qu'elles n'ont pas la structure nécessaire pour assurer les tâches dans le soutien à domicile de personnes âgées en sévère perte d'autonomie nécessitant des soins spécialisés. Les auteurs concluent qu'en général les organisations bénévoles ne peuvent compenser totalement l'absence du réseau informel.

Si la pertinence de la théorie de Litwak est confirmée par certains auteurs, dont Wilcox et Taber (1991), d'autres la remettent en question (Chappell et Blandford, 1991 ; George, 1987 ; Kirwin, 1991 ; McFarland, 1991 ; Noelker et Bass, 1989). Ainsi, George (1987) estime que la perspective théorique de Litwak est «idéale » et que, dans la pratique, les tâches ne se divisent pas toujours d'une façon aussi précise entre les réseaux formel et informel.

\section{Le modèle de Cantor}

Par l'intermédiaire d'une approche holistique et écologique et en s'appuyant sur des données empiriques publiées en 1975, Cantor (1991) a bâti un modèle de système de soin social ( «social care system ») des personnes âgées. Selon elle, le concept de soin social inclut les activités de soutien tant formelles qu'informelles. Le soin social remplit trois types de besoins de la personne âgée, soit 1) les besoins de socialisation, d'affirmation et d'actualisation de soi, 2) les besoins d'assistance dans les tâches de tous les jours (AVD) et finalement 3) les besoins pour les soins de base (AVQ) accompagnant une incapacité fonctionnelle sévère. Le concept de soin social suggère qu'une telle assistance augmente la compétence individuelle de la personne âgée et sa maîtrise de l'environnement. Dans ce modèle, la personne âgée est un acteur important de son propre soutien à domicile. Tout en reconnaissant les différentes provenances du soutien, il met l'accent sur la nature interactive et changeante du système de soin social, dans une perspective à la fois individuelle et écologique. Le soin social comportant des composantes tant formelles qu'informelles, toute tentative de comprendre la façon dont fonctionne le système nécessite un examen des forces individuelles et sociétales ainsi que leurs effets interactifs.

\section{La typologie de Noelker et Bass ainsi que les travaux de Chappell et Blandford}

Dans un article publié en 1989, Noelker et Bass tentent de décrire les différents types de relations ou I'absence de relations qui peuvent s'établir entre les réseaux formel et informel, en fonction des carac- 
téristiques des aînés et de celles des aidants naturels. Chappell et Blandford (1991) ont, quant à elles, examiné plus particulièrement I'interface entre les réseaux d'aide et de soins formel et informel en fonction des conditions particulières des personnes âgées.

À la suite d'une étude menée auprès de plus de 500 aidants naturels, Noelker et Bass (1989) ont construit une typologie permettant de définir les différentes façons par lesquelles les aidants naturels et l'État participent conjointement ou séparément au soutien de la personne âgée à domicile. Les chercheurs ont ainsi dégagé quatre types de relations, soit 1) l'indépendance des proches ou l'absence de relations, 2) la double spécialisation, 3) le lien supplétif et finalement 4) la spécialisation des services formels.

Au cours d'une recherche menée au Manitoba auprès de plus de 1800 personnes âgées, Chappell et Blandford (1991) ont examiné I'indépendance et la complémentarité des réseaux d'aide et de soins formel et informel. Leurs objectifs étaient de mieux comprendre les facteurs liés à I'utilisation ou à la non-utilisation des réseaux formel et informel et de déterminer dans quelles circonstances les réseaux sont complémentaires ou indépendants. Ces auteures ont dégagé différents types de personnes âgées, soit 1) celles qui ne reçoivent aucun service des réseaux formel et informel, 2) celles qui font appel uniquement au réseau informel, 3) celles qui ont recours à la fois aux réseaux formel et informel, quels que soient les types d'aide offerts et 4) celles qui bénéficient de l'aide et des soins provenant des deux réseaux et ce, pour des types d'aide similaires. La contribution de chacun des réseaux est abordée sous I'angle de la dynamique des relations qui se modifient en fonction des caractéristiques de la personne âgée et de ses proches.

En consultant la typologie de Noelker et Bass ainsi que les travaux de Chappel et Blandford, il est apparu intéressant de les présenter simultanément afin de mieux saisir leur spécificité et leur ressemblance. En conjuguant les résultats des deux groupes de chercheurs, six situations ou catégories sont retenues, qui vont de l'absence des réseaux formel et informel à une prise en charge complète d'un des réseaux en passant par la complémentarité de ces deux acteurs. Pour chacune des situations, des éléments de discussion formulés par les deux groupes de chercheurs sont rapportés.

Dans la première situation, il n'y a aucun point de jonction entre les réseaux formel, informel et la personne âgée. La personne âgée est autonome et ne reçoit aucune aide de la part de l'un ou de l'autre de réseaux d'aide et de soins. Selon les travaux de Chappell et Blandford (1991), ces aînés sont généralement moins âgés, moins scolarisés, présentent peu d'incapacités fonctionnelles, ont moins d'amis et vivent seuls. 
Dans la deuxième situation, seul le réseau informel, généralement un membre féminin de la famille, fournit l'aide et les soins à la personne âgée. Noelker et Bass (1989) nomment ce type de situation l'indépendance des proches. Ce type de situation se retrouve particulièrement chez les personnes âgées qui ont peu d'incapacités physiques et qui peuvent compter sur un ou des aidants naturels jouissant d'un état de santé stable. Il peut arriver aussi que la personne âgée et les membres du réseau informel ne connaissent pas l'existence des services offerts par le réseau formel. Chappell et Blandford constatent quant à elles que les personnes âgées qui reçoivent de l'aide uniquement de la part du réseau informel sont habituellement plus jeunes et de sexe masculin. De plus, elles sont généralement moins scolarisées, ont plus d'enfants et cohabitent avec un proche. Sur le plan de la santé, ces personnes ont moins d'incapacités fonctionnelles et moins de conditions chroniques (en nombre et en sévérité) que celles qui reçoivent de l'aide des services publics.

Dans la troisième situation, les réseaux formel et informel fournissent une forme d'aide différente à la personne âgée. Noelker et Bass nomment ce type de relation la double spécialisation. Selon ces chercheurs, la double spécialisation illustre la théorie de Litwak (1985) selon laquelle chaque réseau accomplit les tâches spécifiques qui vont de pair avec sa structure. D'après les travaux de Noelker et Bass, la double spécialisation des tâches entre les aidants naturels et formels se retrouve habituellement chez les aidants naturels de sexe masculin (ex.: le conjoint), chez les aidants naturels vivant un haut niveau de stress associé aux soins et à l'aide à donner à la personne âgée et finalement chez les personnes âgées ayant peu d'incapacités fonctionnelles. Pour leur part, Chappell et Blandford considèrent que la double spécialisation n'a pas d'assises empiriques solides. D'après elles, la recherche à ce jour a montré qu'il n'y a pas de tâches propres à chacun des acteurs engagés dans le soutien à domicile des personnes âgées, comme le prétend Litwak.

La quatrième situation reflète l'engagement des réseaux formel et informel dans des types d'aide similaires. Il s'agit là d'un partage des tâches entre les différents aidants plutôt que d'une spécialisation des tâches. Noelker et Bass qualifient cette relation de supplétive en ce sens que les services formels sont un supplément aux efforts du réseau informel. Les intervenants du réseau public fournissent de l'assistance exclusivement pour des types d'aide et de soins déjà offerts par l'aidant naturel. Par contre, les membres du réseau informel peuvent procurer à la personne âgée des types d'aide et de soins qui ne sont pas fournis par l'État. Les aidants naturels qui rapportent une baisse de l'activité sociale en raison des soins et de l'aide à donner se trouvent 
plus fréquemment dans ce type de relation. De plus, les résultats des travaux de Chappell et Blandford indiquent que les personnes âgées recevant de l'aide de la part des deux réseaux, pour des types d'assistance similaires, ont généralement moins d'enfants, moins de voisins et vivent seules. En d'autres termes, le réseau informel de ces aînés est réduit.

La cinquième situation est caractérisée par un chevauchement partiel des types d'assistance, par une ségrégation partielle des tâches. Les aidants naturels et les aidants formels accomplissent à peu près les mêmes tâches, tandis que les aidants du réseau formel fournissent également de l'aide spécialisée. Noelker et Bass nomment ce type de relation la spécialisation des services formels. Ces chercheurs observent qu'un haut niveau d'incapacité chez l'aîné et un haut niveau de détérioration de la santé de l'aidant naturel sont des prédicteurs de ce type de relation. Chappell et Blandford, quant à elles, concluent que les aînés qui se retrouvent habituellement dans ce type de relation sont plus âgés, plus scolarisés, ont plus d'incapacités fonctionnelles et vivent seuls.

Dans la dernière situation, le réseau formel remplace entièrement l'aide fournie par le réseau informel. Cette relation est qualifiée par Noelker et Bass de substitutive. Ces auteurs mentionnent ne pas avoir trouvé ce type de relation dans leur étude, puisque les personnes âgées ne recevant pas d'aide du réseau informel étaient exclues de leur échantillon.

\section{La typologie de Twigg}

S'inspirant des travaux de Moroney (1986), Twigg (1988) décrit les différents rôles attribués au réseau informel par le réseau formel. Dans une perspective de partage des responsabilités entre la famille et l'État, I'auteure propose trois rôles qui se situent sur un continuum allant d'une contribution limitée de l'État, dans les cas où le réseau informel, généralement la famille, comble la majorité des besoins de la personne âgée, jusqu'à une contribution importante des services formels qui viennent suppléer aux déficits du réseau familial. Ces trois rôles sont : a) les aidants naturels comme ressources, b) les aidants naturels comme exécutants ou coéquipiers et c) les aidants naturels en besoin de ressources ou comme co-clients.

\section{a) Les aidants naturels comme ressources}

Les aidants naturels ne sont pas considérés par le réseau formel; ils existent seulement par rapport à la personne âgée (Moroney, 1986). Dans ce type de relation, la plus grande proportion de l'aide aux aînés est fournie par les aidants informels (Twigg, 1988). Contrairement à d'autres formes de ressources, les aidants naturels 
ne sont pas sujets à la loi de l'offre et de la demande, à la supervision et au contrôle. Les agences de services (réseau formel) font, en quelque sorte, du «résiduel». Elles agissent en considérant que l'informel est une donnée de base, la toile de fond sur laquelle s'organisent les services formels. Dans cette perspective, le focus étant mis sur le client dépendant, le bien-être de l'aidant devient marginal.

\section{b) Les aidants naturels comme exécutants ou coéquipiers}

Dans ce deuxième type de rôle, les réseaux formel et informel partagent l'aide et les soins apportés à la personne âgée. Deux angles sont possibles pour examiner la relation qui unit les aidants naturels et le professionnel. Sous le premier angle, on considère la famille comme membre de l'équipe, mais possédant un statut moindre que celui des professionnels. La famille est perçue comme une extension du professionnel, une exécutante. Le professionnel garde la responsabilité générale du diagnostic et du traitement, mais délègue certaines tâches à la famille, qui remplit les fonctions sous la supervision du professionnel. La seconde prise $d^{\prime}$ angle considère la famille à la fois comme connaissante et agissante. La famille est ici reconnue comme aidant principal. Le professionnel devient un soutien à la famille, et non l'inverse. La famille participe pleinement comme membre de l'équipe et, ainsi, contribue autant à la fonction du diagnostic et de l'évaluation qu'à la phase du traitement.

\section{c) Les aidants naturels en besoin de ressources ou comme co-clients}

Dans ce type de relation, le focus de l'intervention se déplace de l'individu vers la famille. Celle-ci est reconnue comme une unité ayant besoin de soutien pour aider et soigner la personne âgée à domicile. Sans la contribution des services formels, la capacité de la famille à fonctionner à titre d'aidant efficace est menacée et, à la longue, la personne âgée en perte d'autonomie, I'aidant principal et les autres membres de la famille vont en souffrir (Moroney, 1986). Ce type de relation se retrouve généralement dans les situations d'entraide intragénérationnelle (conjoint, frères et sœurs) et lorsque l'aidant naturel a des problèmes de santé qui affectent ses capacités à "prendre soin » (Twigg, 1988). Il existe peu de normes qui définissent clairement quand et pourquoi un aidant naturel peut ou doit être qualifié de co-client. En pratique, la tendance est de tenir compte du fardeau subjectif et d'aider les personnes les plus stressées. Le statut de client demeure toutefois le privilège de la personne âgée. Dans ce type de relation, les aidants naturels deviennent partie intégrante des 
préoccupations du réseau formel en ce qu'ils peuvent contribuer au soutien de la personne âgée.

En résumé, cette théorie, ce modèle et ces typologies présentent les diverses formes de relations ou l'absence de relations qui s'établissent entre les acteurs du soutien à domicile des personnes âgées. Ces différentes relations dépendent notamment des caractéristiques de la personne âgée (âge, niveau d'incapacité, cohabitation, nombre d'enfants, niveau d'instruction), du sexe de l'aidant naturel principal, de son état de santé ainsi que de la disponibilité des autres membres du réseau informel. Les caractéristiques personnelles de la personne âgée et des membres de son entourage sont sujettes à des changements, ce qui introduit l'aspect dynamique évoqué ou sous-entendu par certains auteurs, dont Cantor (1991). Il est important de mentionner qu'aucune donnée empirique provenant de devis de recherche longitudinale ne confirme l'aspect dynamique des contributions des acteurs. Notons de plus que le caractère substitutif du réseau formel qui laisse sousentendre le désengagement des proches dans l'aide et les soins à donner aux personnes âgées à domicile est contesté par plusieurs auteurs (Caro, 1986 ; Chappell, 1985 ; Chappell et Blandford, 1991 ; Edelman et Hughes, 1990 ; Garant et Bolduc, 1990 ; George, 1987 ; Horowitz, 1985 ; Stoller, 1989). Il existe en effet, dans l'état actuel de la recherche, des motifs raisonnables de croire que la présence des services formels $n^{\prime}$ entraîne pas un désengagement des proches mais, tout au plus, une réorientation de leurs activités de soutien (Garant et Bolduc, 1990; Roy et al., 1992). S'il y a une certaine réduction des efforts familiaux lorsque l'État fournit des services, les familles adoptent alors de nouvelles activités de soutien tout aussi importantes pour le bien-être de la personne âgée. L'introduction de services provoque donc un changement de direction des efforts de la famille plutôt que le retrait de celle-ci (Horowitz, 1985).

\section{CONCLUSION}

L'objectif de la présente recension des écrits était d'étudier la dynamique des relations entre les quatre acteurs pouvant être impliqués dans le soutien des personnes âgées vivant à domicile. À la suite de ce travail d'analyse et de synthèse, quelques constats s'imposent.

Au chapitre de la contribution des acteurs au soutien à domicile de la personne âgée, les remarques sont nombreuses. Force est de constater tout d'abord que la personne âgée considérée comme acteur de son propre soutien à domicile est la grande oubliée. Ces résultats contrastent avec le discours du ministère de la Santé et des Services 
sociaux qui situe le citoyen, l'usager au centre de sa politique (Une réforme axée sur le citoyen, MSSS, 1990). Seule Cantor (1991) place la contribution de la personne âgée au centre de son modèle de soin social. En ce qui concerne Jobert (1988), ses propos sont plus une réflexion théorique qu'une mise en application. Il estime que la personne âgée doit être considérée comme un acteur et qu'il faut dépasser les approches qui tentent de faire de cette dernière une consommatrice passive des services. Le succès des politiques de soutien à domicile réside, selon lui, en la capacité de la personne âgée de mobiliser et de combiner les services offerts par la collectivité, ceux qu'elle peut financer elle-même, sa propre capacité de travail domestique et les efforts de son entourage.

Tout comme la personne âgée, les ressources communautaires sont peu étudiées. Sur le plan théorique, elles sont incluses dans le réseau formel. Les recherches québécoises qui distinguent les ressources communautaires et le réseau formel concluent généralement que ces dernières sont peu présentes dans le soutien à domicile des personnes âgées. Encore ici, mentionnons que les ressources communautaires sont un acteur important dans le discours politique (Une réforme axée sur le citoyen, MSSS, 1990).

Une distinction s'impose en ce qui concerne le réseau informel. Ainsi, bien que les auteurs s'entendent pour y inclure l'aide et les soins provenant de la famille, des amis et des voisins, la famille, et plus précisément un membre féminin de la famille, est le principal maître d'œuvre dans le soutien à domicile des personnes âgées. Les amis et les voisins ainsi que les autres membres de la famille, comme aidants secondaires, sont l'exception plutôt que la règle. Dans la littérature actuelle sur le sujet, parler de la contribution des acteurs dans le soutien à domicile des personnes âgées, $c^{\prime}$ est donc parler du partage des responsabilités entre l'État et un ou quelques membres de la famille.

Bien que l'État fournisse, lorsque le besoin se fait sentir, des services spécialisés, particulièrement en matière de soins médicaux, il semble difficile d'établir clairement, selon les conclusions de la majorité des auteurs, quelles tâches appartiennent exclusivement au réseau formel ou au réseau informel, ainsi que le prétend Litwak (1985).

La contribution des réseaux formel et informel, bien qu'elle ne puisse être qualifiée de distincte ou de propre à chacun des réseaux, semble changer, se modifier, en fonction principalement de l'âge et du niveau d'autonomie de l'aîné ainsi que de la disponibilité du réseau naturel, c'est-à-dire généralement la famille. Le caractère changeant de la contribution des acteurs se situe au cœur même de l'objectif général de cette recension, soit l'étude de la dynamique des 
relations. Cette prise d'angle dépasse la simple énumération des tâches effectuées par chacun des acteurs et aborde la contribution de ces derniers dans son aspect à la fois qualitatif (types d'aide et de soins similaires ou différents) et quantitatif (la part d'aide et de soins de chacun par rapport à la somme d'aide et de soins totale procurée).

Dans le but de mieux comprendre les relations ou l'absence de relations entre les acteurs, certains auteurs présentent des théories, modèles et typologies qui, tout en demeurant intéressants, comportent certaines limites. Le postulat à la base de la théorie de Litwak (1985) est que les tâches accomplies par les réseaux formel et informel sont dépendantes de la structure de chaque groupe et, de ce fait, distinctes et propres à chacun des acteurs. L'auteur ne précise pas que ces tâches peuvent être sujettes à des changements, ce qui vaut à sa théorie d'être, selon nous, une théorie "statique " par opposition à une théorie "dynamique » telle que définie dans le cadre de cette recension. Bien que cette théorie reçoive l'assentiment de certains auteurs, elle semble pour plusieurs une vision idéale et théorique plutôt qu'une représentation de la réalité des différents milieux de pratique et des familles avec lesquelles ils travaillent. De plus, la contribution de la personne âgée, tout comme c'est le cas pour d'autres théories et modèles, y est absente.

Le modèle systémique de Cantor constitue, parmi ceux des auteurs recensés, l'apport le plus intéressant quant à la participation des divers acteurs qui gravitent tout près ou en périphérie de la personne âgée, pour lui permettre de demeurer à domicile. La personne âgée est ici un acteur central dans tout le système de «soin social». Les travaux de Cantor reposent sur des données empiriques. L'auteure mentionne, sans toutefois l'avoir mesuré, le caractère changeant de la contribution des acteurs (la dynamique des relations) au soutien de la personne âgée et souligne l'importance d'un devis de recherche longitudinale pour vérifier son hypothèse.

La typologie de Noelker et Bass (1989) ainsi que les travaux de Chappell et Blandford (1991) présentent les relations ou l'absence de relations entre les réseaux formel et informel. Construits sur la base de données empiriques, ces travaux négligent de considérer la personne âgée comme un acteur de son propre soutien à domicile. En combinant les travaux de ces deux équipes de chercheurs, on constate que les six situations présentées offrent une vision détaillée des relations ou de l'absence de relations qui s'établissent entre les réseaux formel et informel. La thèse de la "substitution », c'est-à-dire le désengagement de la famille lorsque le réseau formel fournit de l'aide et des soins, est rejetée par plusieurs auteurs. Le type de relation "supplétive», 
c'est-à-dire la présence des services formels comme supplément aux efforts du réseau informel, semble le plus répandu en contexte québécois, bien que nous ne possédions pas actuellement de données nous permettant de confirmer ou d'infirmer cette hypothèse. De plus, mentionnons que les auteurs décrivent les conditions qui peuvent faire varier les contributions des acteurs sans toutefois mentionner le caractère dynamique des relations.

Nos constats théoriques et méthodologiques sont partagés par certains des auteurs recensés. Ainsi, selon Krout (1985), non seulement peu d'écrits se consacrent aux relations comportant plus de deux acteurs au sein du réseau d'aide de la personne âgée, mais l'examen de l'aspect dynamique et interactif de ces relations est peu présent. Selon Barer et Johnson (1990), I'importance d'une analyse du fonctionnement du réseau de soutien informel en relation avec le réseau de soutien formel a été grandement sous-estimée. Lorsque le rôle d'un seul aidant est mesuré par une liste de tâches, la dynamique de la relation, notentelles, ne peut être comprise.

Ces remarques théoriques et méthodologiques sur l'absence de la personne âgée et des ressources communautaires ainsi que sur le manque de recherche longitudinale pour étudier le caractère dynamique des relations s'adressent aussi à la typologie de Moroney (1986) et de Twigg (1988). Bien que certains auteurs, dont Garant et Bolduc (1990), concluent que le rôle de ressource, plutôt que celui de coéquipier ou de co-client, soit le plus répandu en contexte québécois, ces auteurs négligent de mentionner les données empiriques qui appuient cette conclusion. De plus, comme le soulignent Moroney et Twigg, il est difficile de déterminer par quoi et pourquoi tel aidant naturel est qualifié de co-client. Avec la montée du phénomène de l'entraide intragénérationnelle (aide entre conjoints ou entre frères et sœurs), le portrait des services, en contexte québécois, est changeant. Peut-être que les aidants naturels qui jouent le rôle de ressources aujourd'hui deviendront des co-clients dans un avenir très rapproché. 


\section{Références bibliographiques}

ASSOCIATION POUR LA SANTÉ PUBLIQUE DU QUÉBEC (1988). Le maintien à domicile: à la recherche d'un nouvel équilibre. Compte rendu du colloque. Québec: ASPQ, 110 p.

BARER, B.M. et C.L. JOHNSON (1990). A Critique of the Caregiving Literature, The Gerontologist, vol. 30, $\mathrm{n}^{\circ} 1: 26-29$.

BÉLAND, F. (1982). Les principaux résultats de l'analyse des désirs d'hébergement de trois échantillons de personnes âgées du Québec. Québec: ministère des Affaires sociales, $35 \mathrm{p}$.

BOLDUC, M., L. BÉLANGER et A. DERY (1990). Les services socio-sanitaires aux personnes âgées en perte d'autonomie: la nécessité d'un virage. Québec : ministère de la Santé et des Services sociaux, 18 p.

BRUNET, J. (1987). Rapport du Comité de réflexion et d'analyse des services dispensés par les CLSC. Québec.

CANTOR, M.H. (1991). "Family and Community: Changing Roles in an Aging Society», The Gerontologist, vol. 31, n $3: 337-346$.

CARO, F.G. (1986). «Relieving Informal Caregiver Burden through Organized Services », dans Karl A. Pillemer et Rosalie S. Wolf (dir.), Elder Abuse Conflict in the Family. Dover (Mass.): Auburn House Publishing Company, p. 283-296.

CHAPPELL, N.L. (1987). "The Interface among Three Systems of Care: Self, Informal and Formal », dans Russell A. Ward et Sheldon S. Tobin (dir.), Health in Aging - Sociological Issues and Policy Directions. New York: Springer Publishing Company, p. 159-179.

CHAPPELL, N.L. et A. BLANDFORD (1991). «Informal and formal Care: Exploring the Complementarity », Ageing and Society, vol. 11: 299-317.

COMMISSION D'ENQUÊTE SUR LES SERVICES DE SANTÉ ET LES SERVICES SOCIAUX (1988). Rapport de la Commission d'enquête sur les services de santé et les services sociaux. Québec: Gouvernement du Québec.

COMmISSION ROCHON (1987). Dossier personnes âgées, Programme de consultation d'experts, Commission d'enquête sur les services de santé et les services sociaux. Québec: Gouvernement du Québec.

CONSEIL CONSUltatif NATIONAl SUR LE TROISIÈME ÂGe (1989). Pour mieux comprendre l'autonomie des aînés. $1^{\text {er }}$ rapport: Les obstacles et les avenues de solution. Ottawa: ministère des Approvisionnements et Services.

CONSEIL CONSULTATIF NATIONAL SUR LE TROISIÈME ÂGE (1990). Vieillir et le CCNTA. La position du CCNTA sur les services communautaires dans les soins aux aînés. Ottawa: ministère des Approvisionnements et Services.

EDELMAN, P. et S. HuGHES (1990). "The Impact of Community Care on Provision of Informal Care to Homebound Elderly Persons», Journal of Gerontology: Social Sciences, vol. 45, $\mathrm{n}^{\circ} 2$ : S74-S84.

FACTOR, A.R. (1987). The Frail Elderly's Use of Homemaker Services in Conjunction with their Natural Support Networks. Chicago: University of Illinois, $245 \mathrm{p}$.

GARANT, L. et M. BOLDUC (1990). L'aide par les proches: mythes et réalités. Québec: ministère de la Santé et des Services sociaux, Direction de l'évaluation, Collection Études et analyses, $\mathrm{n}^{\circ} 8: 160 \mathrm{p}$. 
GeOrGE, L.K. (1987). «Easing Caregiver Burden: The Role of Informal and Formal Supports », dans Russell A. Ward et Sheldon S. Tobin, Health and Aging - Sociological Issues and Policy Directions. New York: Springer Publishing Company, p. 133-158.

GRANDMAISON, A. et C. GOSSELIN (1986). Évaluation des soins et services à domicile, des soins de répit et des ressources de protection pour les personnes âgées. Montréal: Centre des services sociaux du Montréal métropolitain, $100 \mathrm{p}$.

HARPER, S. et D.A. LUND (1990). "Husbands and Daughters Caring for Institutionalized Dementia Patients : Toward a Model of Caregiver Burden», International Journal of Aging and Human Development, vol. 30, $\mathrm{n}^{\circ} 4: 241-262$.

HOKENSTAD, M.C. et L. JOHANSSON (1990). "Caregiving for the Elderly in Sweden - Program Challenges and Policy Initiatives», dans David E. Biegel et Arthur Blum, Aging and Caregiving - Theory, Research and Policy. Newbury Park: Sage Publications, p. 254-269.

Horowitz, A. (1985). "Family Caregiving to the Frail Elderly », dans M. Powell Lawton et Georges L. Maddox (dir.), Annual Review of Gerontology and Geriatrics, vol. 5, p. 194-246.

HuGHES, S.L. et M. GUIHAN (1990). "Community-Based Long Term Care: The Experience of the Living At Home Programs », Journal of Gerontological Social Work, vol. 15, no 3-4: 103-129.

JOBERT, B. (1988). "Action publique et solidarité civile: le cas du maintien à domicile des personnes âgées », Revue internationale d'action communautaire, vol. 59: 89-93.

Joubert, P., A. LAberGe, J.-P. Fortin, M. PARAdis et F. Desbiens (1991). Évaluation du programme de services intensifs de maintien à domicile (SIMAD). Sainte-Foy: Unité de recherche en santé communautaire, Centre de recherche du CHUL.

JUTRAS, S. et F. VEILLEUX (1989). Des «partenaires» méconnus: les aidants des personnes âgées en perte d'autonomie. Montréal: Université de Montréal, Groupe de recherche sur les aspects sociaux de la prévention, $109 \mathrm{p}$.

KIRWIN, P.M. (1989). An Examination of the Relationship between Formal and Informal Systems in the Service of the Adult Day Care for the Frail Elderly. Bryn Mawr College, The Graduate School of Social Work and Social Research, 259 p.

KIRWIN, P.M. (1991). Adult Day Care. The Relationship of Formal and Informal Systems of Care. New York et Londres: Garland Publishing, 238 p.

KROUT, J.A. (1985). "Relationship Between Informal and Formal Organizational Networks », dans William E. Sauer et Raymond T. Coward, Social Support Networks and the Care of the Elderly. New York: Springer Publishing Company, p. 178-195.

LITWAK, E. (1985). Helping the Elderly, the Complementary Roles of Informal Networks and Formal Systems. New York: The Guilford Press, 306 p.

LITWAK, E., P. MESSERI et M. SILVERSTEIN (1990). "The Role of Formal and Informal Groups in Providing Help to Older People», Marriage and Family Review, vol. 15, $\mathrm{n}^{\circ}$ 1-2: 171-193. 
MCFARLAND, M.L. (1991). The Effect of the Provision of in-Home Services on the Elderly Person's Informal Support Networks. Baltimore: University of Maryland, $217 \mathrm{p}$.

MINISTÈRE DE LA SANTÉ ET DES SERVICES SOCIAUX (1990). Une réforme axée sur le citoyen. Québec: Gouvernement du Québec.

MINISTÈRE DE LA SANTÉ ET DES SERVICES SOCIAuX (1992). La politique de la santé et du bien-être. Québec: Gouvernement du Québec.

MORONEY, R.M. (1986). Shared responsability - Families and Social Policy. New York: Aldine Publishing Company, 218 p.

NOELKER, L.S. et D.M. BASS (1989). "Home Care for Elderly Person: Linkages Between Formal and Informal Caregivers », Journal of Gerontology, vol. 44, $\mathrm{n}^{\circ} 2:$ 563-570.

PANET-RAYMOND, J. et A. VANDAL (1991). "Le maintien à domicile à I'heure du partenariat entre organismes communautaires et CLSC », Le Gérontophile, vol. 13, $\mathrm{n}^{\circ} 3: 19-22$.

PRUCHNO, R.A., J.E. MiChAELS et S.L. POTASHNIK (1990). «Predictors of Institutionalization among Alzheimer Disease Victims with Caregiving Spouses », Journal of Gerontology, vol. 45, $\mathrm{n}^{\circ} 5$ : 259-266.

ROY, J., A. VÉZINA et M. PARADIS (1992). De la tâche à la mission. Personnes âgées, aidants naturels, services intensifs de maintien à domicile (SIMAD). Sainte-Foy, Centre de recherche sur les services communautaires, Université Laval.

SANTÉ ET BIEN-ÊTRE SOCIAL CANADA (1986). Vieillir: Vers une nouvelle perspective. Ottawa: Division de la promotion de la santé.

SCHIRM, V.L. (1987). Shared Caregiving by Formal and Informal Caregivers of Community Residing Elderly. Case Western Reserve University, 173 p.

Seltzer, M.M., J. IVRY et L.C. LitCHField (1987). "Family Members as Case Managers: Partnership between the Formal and Informal Support Networks », The Gerontologist, vol. 27, $\mathrm{n}^{\circ} 6:$ 722-728.

SOLDO, B.J. et al. (1989). "The Balance Between Formal and Informal Care», dans Marcia G. Ory et Kathleen Bond, Aging and Health Care - Social Science and Policy Perspective, Londres et New York: Routledge, p. 193-216.

STOLLER, E.P. (1989). "Formal Services and Informal Helping: The Myth of Service Substitution », Journal of Applied Gerontology, vol. 8, no 1 : 37-52.

TWIGG, J. (1988). «Models of Carers: How Do Social Care Agencies Conceptualize Their Relationship With Informal Carers? », Journal of Social Policy, vol. 18, $\mathrm{n}^{\circ} 1:$ 53-66.

WILCOX, J. A. et M.A. TABER (1991). "Informal Helpers of Elderly Home Care Clients », Health and Social Work, vol. 16, n 4: 258-265.

WiSENDALE, S.K. (1983). Home Care of the Elderly: Household Composition as a Predictor of the Need for and Utilization of Formal and Informal Services by an Elderly Population. Implications for Long-term Care Policy. Brandeis University, $376 \mathrm{p}$. 